\title{
Population et structures professionnelles à Bruges aux XIVe et
} $\mathrm{XVe}$ siècles

Jan Dumolyn

\section{Citer ce document / Cite this document :}

Dumolyn Jan. Population et structures professionnelles à Bruges aux XIVe et XVe siècles. In: Revue du Nord, tome 91, n³29, Janvier-mars 1999. pp. 43-64;

doi : https://doi.org/10.3406/rnord.1999.2905

https://www.persee.fr/doc/rnord_0035-2624_1999_num_91_329_2905

Fichier pdf généré le 02/11/2020 
Bevolking en beroepstructuur te Brugge in de 14de en 15de eeuw.

Zoals algemeen geweten vormden de late middeleeuwen een turbulente periode voor de stedelijke economieen in Vlaanderen. De aanwijzingen over het bevolkingsaantal van de wereeldmarkt Brugge, waarover we op drie precieze momenten beschikken (1338-1340, 1440, 1477 ), suggereren een relatieve stabiliteit tijdens de 14 de en 15 de eeuw. Dit aantal fluctueerde tussen ongeveer 30000 tot 40000 inwoners. De demografische beweging volgde ongeveer de conjunctuur eigen aan het «Bourgondisch model». Mortaliteitscrissisen werden afgewisseld met periodes van heropbloei, waarbij de immigratie de voornaamste factor was. De vergelijking tussen militaire gegevens voor de jaren 1338-1340 en 1436 geeft ons een idee over de socioprofessionele structuren en bevestigt het algemeen beeld van de industriele herstructureringen in het laatmiddeleeuwse Brugge. De groei van de luxe-industrie en de confectienijverheid konden het verlies aan werkgelegenheid in de textielnijverheid echter niet compenseren.

\begin{abstract}
Population and Professional Structures in Bruges in the XIVth and XVth centuries.

It has been widely recognized that, with regard to the Flemish urban economies, the Lower MiddleAges were an age of disturbance. The indications that we possess about the number of inhabitants in Bruges, the major European market, fort three precise periods (1338/1340, 1440, 1447), suggest a relative stability through the XIVth and XVth centuries, the population fluctuating between 30,000 and 40,000 people. The demographic movement followed more or less closely the circumstancial variation of the «Burgundian economic model». Periods of high death rates were followed by periods of recovery, in which immigration played a dominant part. The comparison between the military data regarding the years 1338 and 1436, which gives us an idea of the socio-professional structures, confirms the general picture of the industrial restructurations taking place in Bruges in the Lower Middle- Ages. The growth of the luxury and garment-making industries was not however sufficient to compensate the jobs lost in the cloth-making industry.
\end{abstract}

\title{
Résumé
}

Il est bien connu que, pour les économies urbaines flamandes, le Bas Moyen Age fut une période turbulente. Les indications du nombre d'habitants de Bruges, premier marché européen, dont on dispose à trois moments précis $(1338-1340,1440,1477)$, suggèrent une stabilité relative aux XIVe et $X V$ e siècles, fluctuant entre environ 30000 et 40000 habitants. Le mouvement démographique suivait plus ou moins le mouvement conjoncturel du " modèle économique bourguignon ». Des crises de mortalité étaient suivies par des périodes de redressement, dans lesquelles l'immigration était le facteur principal. La comparaison entre les données militaires des années 1338-1340 et 1436, qui nous donne une idée des structures socioprofessionnelles, confirme l'image générale de restructurations industrielles à Bruges au Bas Moyen Age. La croissance de l'industrie de luxe et de la confection ne pouvait cependant pas compenser les pertes d'emploi dans la draperie. 


\section{Population et structures professionnelles à Bruges aux $X^{2} V^{e}$ et $X V^{e}$ siècles}

\section{La population brugeoise au bas Moyen Age : état de la question*}

Il y a quinze ans, W. Prevenier a publié dans la Revue $d u$ Nord une synthèse provisoire sur la démographie des villes flamandes au bas Moyen Age'. Bruges y figure comme deuxième ville du comté avec une population estimée de 36738 à 45921 habitants en 1338/1340. Une mise au point récente pour toutes les villes flamandes médiévales n'ajoute rien à cet état des connaissances $^{2}$. Comme dans bien d'autres cas, les chiffres démographiques brugeois pour le XIV" siècle sont basés sur le nombre d'hommes armés dans les milices communales. A Bruges ce sont les campagnes militaires de 1338-1340 l'époque de Jacques d'Artevelde, gouverneur de Flandre et meneur populaire - qui nous ont fourni ces chiffres ${ }^{3}$. Il n'est pas nécessaire de répéter tous les arguments méthodologiques difficilement contestables, qui ont donné les chiffres, retenus par Prevenier, de 33580 habitants minimum et 41975 habitants maximum, résultat d'une multiplication du nombre de chefs de familles par les coefficients 4 et $5^{4}$. Le chiffre réel serait plus proche du maximum. Il

* - Jc tiens d'abord à remercier lc professeur M. Boone et le docteur P. Stabcl pour leur remarques, et le professeur Th. de Hemptinne pour la correction du texte

1. - W. Prevenier, « La démographie des villes du comté de Flandre aux XIV" et XV" siècles. Etat de la question. Essai d'interprétation », Revue du Nord, 1983, p. 255-275; une version plus élaborée a été publiée en néerlandais : "Bevolkingscijfers en professionele structuren der bevolking van Gent cn Brugge in de 14de eeuw ", dans Album Charles Verlinden, Gand, 1975, p. 264-303; voir aussi la synthèse pour l'ensemble des Pays-Bas dans le Nieuwe Algemene Geschiedenis der Nederlanden, t. IV, Bruxelles, 1980.

2. - P. Stabel, Dwarfs among giants. The Flemish Urban Network in the late Middle Ages, Leuven-Apeldoorn, 1997, p. 32. Voir aussi les actes de la conférence « Le réseau urbain en Belgique dans une perspective historique (1350-1850). Une approche statistique et dynamique », Bruxelles, Crédit communal de Belgique, 1992 et notamment les contributions de W. Prevenier, J.-P. Sosson, M. Boone et P.M.M. Klep, laissant de côté les chiffres absurdes et complètement fantaisistes donnés par P. BaIroCH, J. B NIOU et P. CIIEvre, La population des villes européennes. Banque de données et analyse sommaire des résultats 800-1850, Genève, 1988 (125000 habitants à Bruges en 1400 !).

3. - J.-F. VeribrugGen, Het gemeenteleger van Brugge van 13.38 tot 1340 en de namen van de weerbare mannen, Bruxelles, Koninklijke Commissie voor Geschicdenis, 1962: c'est l'édition du Register van herevaerden (registre des campagnes militaires).

4. - W. PrHVhenilik, « Bevolkingscijfers... », p. 281-283. Sclon R. MOLS, Introduction à la démographic historique des villes d'Europe du XI'" au XvIr' siècle, t. II, 1955, p. 100-109 le coefficient de 5 est préférable pour les villes avant le XvI" sic̀cle ct Prevenier ajoute (art. cit., n. 31) que cela cst 
ajoute 3946 personnes qui n'étaient pas comprises dans les cadres officiels des métiers ou de la bourgeoisie, et parvient ainsi à un total de 36738 (minimum) à 45921 (maximum) habitants 5 .

De Meyer a compté les redevables de la capitation de 1394-1396 pour trois des six sections de la ville ou zestendelen : 1395 dans la section de SaintJacques, 1169 dans la section de Saint-Nicolas et 1087 dans la section de Notre Dame. Il n'a pas été possible de déterminer par extrapolation le nombre de redevables pour les trois autres sections. Utilisant le coefficient 4,9 (selon elle habituel pour les villes commerciales), De Meyer parvient à un résultat de 17914 habitants pour la moitié de la ville ou, multiplié par deux : 35770 personnes; c'est donc la quasi-stabilité entre 1338 et 1394'! Evidemment, il est peu probable que ces trois des six zestendelen représentaient exactement la moitié de la population! En outre il s'agit ici de listes de contribuables, qui sont en règle générale plus complètes que les recensements d'hommes valides. Après avoir uniformisé les chiffres en remplaçant le coefficient 4 par 5 pour homogénéiser les calculs, afin de faire une comparaison valable, Prevenier ajoute environ 1000 personnes jouissant de l'immunité fiscale étrangers, nobles, clercs et officiers du prince ; le résultat serait alors 37510 (coefficient maximum : 5), c'est-à-dire un modeste déclin comparé aux 45921 de $1338-1340^{7}$.

La question se pose alors de l'influence de la décadence portuaire de Bruges sur la population et les structures socioprofessionnelles au $\mathrm{XV}^{\mathrm{e}}$ siècle. Cette régression a attiré l'attention de beaucoup d'historiens. Parmi les explications traditionnelles figurent : des problèmes hydrographiques (l'ensablement du Zwin), le déclin de la draperie flamande et l'essor des nouvelles draperies en Brabant, Hollande et Angleterre, le commerce trop réglementé et dominé par les courtiers et hôteliers, des problèmes avec la Hanse teutonique, des facteurs politiques, le développement du marché anversois ${ }^{8}$ etc.

\footnotetext{
4. - (suite) surtout le cas pour une ville commerciale comme Bruges dù les familles moyennes étaient plus riches el plus larges que dans une ville industrielle (pour Gand il préfere une multiplication par 4). A. Di:Rvillti. " Le nombre d'habitants des villes d'Artois el de la Flandre wallonne (1300-1450) ». Revele de Nord, 1983, p. 280-281. prélère un coefficient de 3.5 pour une ville industrielle: d'autres exemples de la variation de ce coefficient dans J. Hlis:Rs, "Les limites des méhodes statistiques pour les recherches de la démographie médiévale ". Annalés dé Démographie Historique, 1968. p. 50-53 et A. Hicol:NET-NADAl. "I a démographie des villes françaises alu Moyen Age », Amuale's de Démegraphie Historicute, 1980, p. 209.

5. - W. PRt:Vi:VItR. "La démographie... », p. 284.

6. - I. DI: Mtrier. " De sociale strukturen te Brugge in de Ite ecuw », dans W. Btockmass e.a.

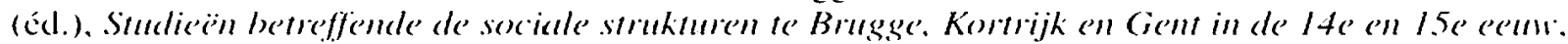
Kortrijk-Heule. 1971. p. 7-78 (Aneiens Pays el Assemblées d'Elats, I.IV).

7. -- W. PrivienII:R, « Lal démographic... », p. 289-290.

8. - Ies synthèses réeentes les plus importantes sont: J.A. VA.: HolTrt: "The rise and decline of the market of Bruges ", Economic History Review, 1966, p. 29-47; W. BRtiLl:. "Brugge en Antwerpen in de 15de en lode ceuw, een tegenstelling? ". Tijdschrift vor Geschiedenis, 1970, p. 15-37, traduit dans les Acta Historiae Neerlandicae, 6. 1973, p. 1-26: M.K.E. GotTschal.k.
} 
Toutefois, dans la période que nous envisageons — de 1340 à 1440 environ - ce déclin n'avait pas entore commencé, ou du moins n'était-il pas perçu par les contemporains. Malgré la bibliographie considérable sur le sujet, Van Uytven constate que la chronologie et les causes du déclin n'ont pas encore été suffisamment étudiées, par manque de preuve quantifiable.

En 1438, il ne faisait encore aucun doute pour Philippe le Bon que Bruges était la ville la plus renommee par tout le monde par le fait de marchandise". Les réévaluations du «Transport de Flandre », c'est-à-dire la répartition des aides (ou subsides) au comté de Flandre par les différentes villes et circonscriptions rurales, donnent une vue relativement stable de la position économique de Bruges dans le comté (aussi bien du point de vue de la démographie que de la richesse des habitants). Au début du XIV $V^{0}$ siècle, Bruges payait $15,2 \%$ des aides flamandes, en $140815,7 \%$ et en 1517 encore $14,4 \%{ }^{10}$. Le revenu annuel du grand tonlieu du Zwin était de $7800 \mathrm{lb}$. par. en 1384 et de 8400 lb. par. en 1432 et en 1440 . Le premier véritable signe de déclin s'aperçoit en 1464 (6800 lb. par.) et en 1487 on n'atteint qu'un revenu de $6100 \mathrm{lb}$. par." . Ce n'est qu'à partir de 1450 que plusieurs nations de marchands étrangers commencent à quitter la métropole et c'est seulement vers la fin du siècle que leurs absences deviennent plus longues, voire définitives ${ }^{12}$.

Pour 1477, on dispose du chiffre de 42000 habitants calculé par A. Janssens ${ }^{13}$. C'est le résultat d'un calcul parfois osé, mais ingénieux et globalement acceptable sur le plan méthodologique. Une analyse minutieuse des comptes de la ville a permis à l'auteur d'aboutir au chiffre de 1299 miliciens des corporations et de la bourgeoisie, mobilisés contre la menace française en mai $1477^{14}$. Il y ajoute environ 100 personnes qui ne ressortiraient d'après lui ni de la bourgeoisie, ni des corps de métiers, comme les huissiers, sergents et

8. - (suite) « Het verval van Brugge als wereldmarkt », Tijdschrift voor Geschiedenis, 1953. p. 1-26; W. Blockmans, «Brugge als Europees Handelscent rum » dans : V. VermeERSCH (éd.), Brugge en Europa. Antwerpen, 1992, p. 40-56 et R. VAN UYTVEN. « Stagcs of cconomic decline : late medieval Bruges », dans Peasants and townsmen in medieval Europe. Stadia in honorem Adriaun Verhulst, Gent, 1995, p. 259-270) voir aussi J.-P. Sosson, « Corporation et paupérisme aux $X I V^{*}$ et $X V^{*}$ siècles. Le salariat du bâtiment en Flandre et en Brabant et notamment à Bruges », dans Tijdschrifi voor Geschiedenis, 1979, p. 557-575 ct ID., Les travaux public.s de la ville de Bruges. XIW et $x$ V siècles: les matériaux, les hommes, Bruxelles, 1977 pour des donnécs conjoncturclles.

9. - R. VAN UYTVEN, « Stages of economic declinc... », p. 258-259; la citation est de Gili.IODTS VAN SeVEREN, Inventaire analyique des archives de la ville de Bruges, t. V, p. 576.

10. - R. VAN UYTVI:N, ibideim, p. 261-262.

11. - R. VAN UYTVEN, « La Flandre et le Brabant, « terres de promission » sous les ducs de Bourgogne? ", Revue du Nord, 1961, p. 282.

12. - J. MARl:CHAI., «Le départ de Bruges des marchands étrangers, Xve el Xvi" siècle », Annales de la Société d'Emulation de Bruges, 1951, p. 27 el passim.

13. - A. Janssi:ns, "Het Brugse bevolkingsaantal in 1477 ", in Van Middeleeumen tor heden. Bladeren door Brugse kunst en geschiedenis, Bruges, 1983, p. 29-35.

14. - W. Blockmans el W. Pri:vinitir, Les Payis-Bas bourguignons, p. 206. 
clercs de la ville, ce qui n'est absolument pas certain ${ }^{15}$. Quand on sait qu'un homme sur sept était convoqué, on parvient selon le coefficient 4 ou 5 à respectivement 36772 et 45962 habitants. En plus, il ajoute 1000 étrangers, 500 religieux et 74 pauvres pour finalement arriver à une moyenne de plus ou moins 42000 habitants entre les limites de 38346 et $47536^{16}$. Le nombre d'étrangers semble acceptable, tenant compte du fait que les grands marchands avaient un petit personnel nombreux et que les riches Brugeois disposaient parfois de domestiques français, brabançons etc. Toutefois, ce nombre variait constamment et manifestement selon la saison ${ }^{17}$. 500 religieux nous paraît être un minimum pour une ville où il y avait entre 25 et 30 monastères, abbayes et béguinages, environ 10 églises dont deux églises collégiales, environ 15 chapelles et 10 institutions charitables importantes ${ }^{18}$. Intégrer 74 pauvres dans le calcul, cela paraît bien sûr une absurdité. L'auteur se base sur une dépense, mentionnée dans les comptes de la ville de 74 habits pour les dullen, vondelinghen ende aerme lieden van der stede («Les déments, les enfants trouvés et les pauvres gens de la ville $»)^{19}$. Sans doute s'agit-il d'un petit nombre de malheureux sélectionnés par la ville pour bénéficier d'aumônes. La quantité réelle de pauvres non-brugeois qui habitaient ou fréquentaient souvent la ville était sans doute beaucoup plus considérable. Comme

15. - A. JANSSENS. « Brugse bevolkingsaantal... », p. 31-32. Il nous semble au contraire plus acceptable que tout homme non-inscrit dans un des 54 métiers brugeois, ressortissait du membre de la bourgeoisic. Sinon, comment expliquer le nombre d'hommes relativement grand fourni par ce membre? Il est difficile de croire que ceux-ci étaient tous de riches marchands, mêne s’il fallait payer son propre armement.

16. - Ibidem, p. 32.

17. - J.A. VAN HoUTTE, «Handel en nijverheid", in Nieuwe Algemene Geschiecienis van de Nederlanden, t. IV, p. 90, cite la chronique de Nicolas Despars (éd. J. de Jonghe, Bruges-Rotterdam, 1842. t. III, p. 431-432) qui compte 136 Hanséates, 150 Italiens et 48 Espagnols at cortège de 1440) à l'occasion de la première visite du duc après la répression de la révolte de 1436-1438. Van Houtte remarque qu'il y avait encore d'autres nations à Bruges qui ne sont pas mentionnées par Despars et que ce cortège était organisé au mois de décembre, à un moment où il y avait normalement peu de marchands étrangers dans la ville. A la saison d'affaires, I000 étrangers résidant à Bruges lui semble plausible: cf. aussi W. Paravicini, "Bruges and Germany », in V. Vhermiersch (éd.), Bruges and Furope, Anvers, 1992, p. 1001-114.

18. - M. RycKatrT, Historische stedenatles van België, Bruges-Bruxelles, 1991, p. 113 et passim. A. JANssiss, « Brugse bevolkingsaantal... ", p. 32 a compté environ 160 clercs dans les comptes de la ville. qui ne devaient pas payer la taxe sur la bière, mais ici les réguliers manquent presque totalement et il n'est pas du tout certain que tous les séculiers soient compris. J. WAric lH\%., "Etat bénéficial de la Flandre et du Tournaisis au temps de Philippe le Bon (1455) ", Analectes pour servir à l'hisforie écelésiastique de la Belgique, 3" séric, t. V, 1909, p. 440-452 mentionne cnviron 110 cleres séculiers à Bruges: cependant. tenant compte de lécart entre ce chiffre plutôt théorique et le chiffre récl qu'ont constaté M. BOONE et Th. DE HiMPTINNI, « L_e clergé séculier gantois en 1498-1499 », Bulletin de la Commission Rovale d Histoire, 1983, p. 377-430, nous proposons 200 séculiers dans celle ville riche. W. Simons. Stad en Apostolaat. De vestiging van de bedelorden in het graafschap Vlaanderen (ca. 1225-ca.1350), 1987, Bruxelles, p. 150 : environ 270 membres masculins d'ordres mendiants en 1340; ce même auteur a bien voulu nous communiquer ses estimations pour les autres réguliers et les béguines (sur lesquelles il prépare une nouvelle étude) el bogards ; après cette discussion on propose environ 1000 à 1200 " cleres » brugeois.

19. - A. JANSSENS, « Brugse bevolkingsantaal... », p. 32 ; Comptes de la ville 1480-1481, fol. $61 \mathrm{r}^{\circ}$. 
c'est presque toujours le cas pour les villes médiévales, il n'existe aucune source pour calculer ce nombre et même un instantané statique nous dirait peu sur ce phénomène dynamique, aussi bien lié à la conjoncture de l'économie rurale qu'à celle de l'économie urbaine. Dans les villes médiévales et d'Ancien Régime les taux de mortalité surpassaient toujours ceux de natalité. Mais d'autre part une immigration considérable et presque constante assurait la stabilité ou même une croissance démographique. Pour la Flandre, les possibilités d'emploi dans les villes étaient le pull-factor démographique. comme la surpopulation marquée dans des zones rurales souvent proto-industrialisées constituait le push-factor. Ces fluctuations étaient aussi déterminées par les crises et disettes et l'évolution des salaires réel $\mathrm{s}^{20}$. La société relativement avancée des Pays-Bas bourguignons voyait un va-et-vient intense entre ville et campagne et entre les différentes villes ${ }^{21}$. En moyenne, 3 à 3,5 nouveaux bourgeois sur 1000 achetaient chaque année le droit de bourgeoisie dans les villes médiévales des Pays-Bas méridionaux ${ }^{22}$. Ainsi, entre 1337 et 1371 , il y avait une immigration « officielle » à Bruges d'environ 143 personnes par an, mais rien ne suggère que ceci faisait croître la population ${ }^{23}$. Entre 1418 et 1450, 197 nouveaux venus s'inscrivaient annuellement dans les registres de bourgeoisie; 142,2 entre 1456 et 1478 et - pendant la chute démographique à la fin du siècle - 84, 6 entre 1479 et $1486^{24}$. Selon David Nicholas, dans les années de mortalité moyenne (sans trop d'excès causés par une épidémie), l'immigration à Bruges suffisait pour compenser l'excédent

20. - P. STABEL, Dwarfs among Giants..., p. 116-117; II)., De kleine stad in Vlaanderen. Bevolkingsdynamick en economische functies van de kleine en secundaire stedelijke centra in het Gentse kwartier (14de-16de eetuw), Bruxelles, 1995, p. 54-61; Un aperçu des problèmes méthodologiques pour l'étude de l'immigration urbaine en Flandre dans E. THOEN, Landbouweconomie en bevolking in Vlaanderen gedurende de late Middeleeuwen en het begin van de Moderne Tijden. Testregio : de kasselrijen van Oudenaarde en Aalst, Gand, 1988 p. 189-196 (Centre belge d'histoirc rurale, $\mathrm{n}^{\circ} 90$ ).

21. - W. Blockmans, W. Pri:vi:nilik, G. Pil:tFrs et R.W.M. Van Scilaïk. «ussen Crisis en Welvaart », dans Nieuwe Algemene Geschiecienis der Nederlanden, I. IV, p. 52. On a depuis longtemps abandonné l'image « juridiste » d'un fort antagonisme entre ville et campagnc.

22. - P. STABlil, De kleine stad..., p. 39.

23. - A. JAMEES, Brigse poorters. Opgetekend wit de stadsrekeningen, 1. I, 1281-1417, Handzame, 1974. p. XXXIII-XXX: à Ypres c'était la même situation : H. PIRENNE, « Les dénombrements de la population d'Ypres au Xv" siècle », Vierteljahrschriff fïr Sozial-und Wirschaftsgeschichte, 1, 1903, p. 458-489.

24. - J. A. VAN Houml, De geschiedenis van Brugge, Ticlt, 1982, p. 418; R. A. PArmintirir, Indices op de Brugse poortersboeken, 2 t., Bruges, 1938. Des chiffres moyens par décennic dans : E. THOEN, «Immigration to Bruges during the late Middle Ages», in Le Migrazioni in Europa secc. XIII-XVIII. Atti della "20a Settimana di Studi »3-8 maggio 1993, Prato, Istituto Internazionale di Storia Economica «Franccsco Datini », 1994, p. 337, surtout table 1; voir aussi M. CarLIER, «Migration Trends in the Towns of Flanders and Brabant (15th-18th Century) », ibidem, p. 355-370 et la synthèse à ce sujet dans Ph. DOLLINGER, « Les recherches de démographie historique sur les villes allemandes au Moyen Age ", in La démographie médiévale. Sources ét méthodes. Actes du congrès de l'Association des Historiens Médiévistes de l'Enseignement Supérieur public (Nice. 15 au 16 mai 1970), Nicc. 1972, p. 115-116. 
de mortalitées. Il est vrai que les registres aux bourgeois nous montrent seulement l'immigration d'ouvriers qualifiés et d'autres éléments des classes moyennes, mais d'autre part on ne peut pas nier que ceux-ci forment un groupe crucial sur le plan économique ${ }^{26}$. Leur nombre constitue donc un bon indicateur de la vitalité économique de la ville et suggère que le déclin économique de Bruges à la fin du XIV“ siècle fut temporaire et qu'il était déjà surmonté dans la deuxième décennie du $\mathrm{XV}^{6}$ siècle $^{27}$. Nous pensons pouvoir confirmer globalement cette hypothèse d'E. Thoen en calculant la population brugeoise en 1436-1441. Mais donnons d'abord une idée des structures socio-économiques de la ville de Bruges du bas Moyen Age.

\section{Répartition professionnelle, 1338-1436}

Le voud était l'unité de base de la milice urbaine brugeoise, qui fixait le nombre de miliciens que les corps de métiers et la bourgeoisie devaient fournir. Le voud de 1316 comptait exactement 600 miliciens, celui de 1338-1340, 607 personnes $^{2 x}$. Dans ce dernier il y avait, par exemple 96 tisserands, 1 plombier, 14 maréchaux et 96 bourgeois. Les miliciens de la bourgeoisie étaient divisés en 6 zestendelen ou sections de ville. On peut supposer que ces chiffres correspondaient plus ou moins à l'importance démographique des corporations et des bourgeois et qu'ils nous donnent une idée de la répartition socioprofessionnelle. On voit cependant que, pour 53 des 56 corporations brugeoises, la répartition du nombre d'hommes à fournir en 1338-1340, était restée inchangée en $1379^{20}$. Si ces chiffres avaient pu correspondre à une réalité démographique au moment de leur fixation en 1338, ils étaient complètement sclérosés en 1379 et ce serait une erreur méthodologique de les utiliser

\footnotetext{
25. - D. Nicholas. Fonn and Comntryide. Social. economic and political Tensions in Fonte'enthC'nfury Flanders. Bruges. 1971 , p. 23.5.

26. - P. StABia.. Du'arfs among giants..., p. 128.

27. - E. Thotiv. « Immigration 10 Bruges... ». p. 353 : cet atueur n’a cependant pas assez lenu compte des changements dans les tarifs de bourgeoisie, voir W. BI.oCK.MANS. "The creative environment. Incentives $t o$ and functions of Bruges Art Production 》 dans : M.W. AlvsworTh (éd.),

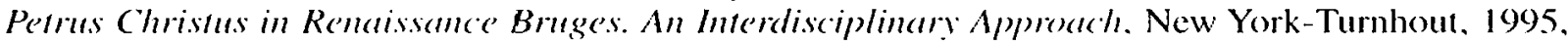
p. 13.19.

28. - J.-F. VERbrlicicilin. " De organisat ie van de militic te Brugge in de XIVde ecuw ", Ammales de"

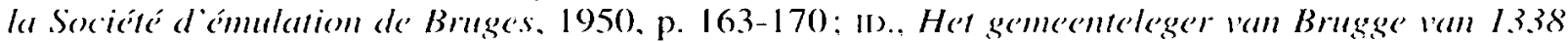

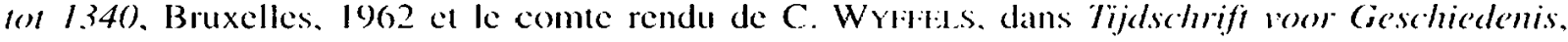
196.3. qui ajoute une correction importante.

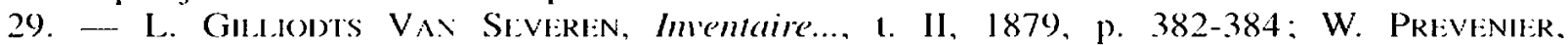
Berolkingsciffers..., p. 289. Dans ee cas il sagit du paiement du moendghelt, une solde mensuelle pour la milice urbaine. Cette solde apparaît pour la première fois dans les comptes de la ville en 1349-1350 et fut payée sans interruption jusqu’à 1382. date de la défaite des révolutionnaires brugeois après la bataille de Rozebeke. et fut ensuite supprimée. Après l’action réussie des corps de métiers brugeois en 1411 (voir plus loin), elle fut de nouveau payée, mais seulement pour deux mois. En 1436, on décida de recommencer le paiement au cours des préparatifs pour l'expédition de Calais. Après la répression de la révolte brugeoise de 1436-1438. le due ordonna la suppression de

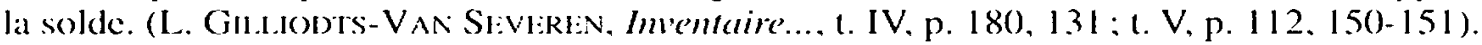


pour étudier la répartition professionnelle à Bruges à la fin du XIV ${ }^{\circ}$ siècle. Pour cette époque on dispose d'autres chiffres, provenant des listes de redevables de la taille urbaine, conservées pour trois des six zestendelen des années 1394-1396.31. Il s'agit d'une pointinghe ou capitation personnelle sur la fortune de chaque chef de famille. Les redevables ont été identifiés dans d'autres sources pour déterminer autant que possible leurs occupations et leur statut social. Quoiqu'on ne puisse pas les mettre en parallèle avec les données des voud, il est toutefois possible de calculer des chiffres qui donnent une idée de l'importance respective des différents secteurs économiques dans une moitié de la ville. I. de Meyer a exhaustivement analysé les données qu'elle a répertoriées et elle a aussi comparé les chiffres de 1302, 1338-1340, 1379 et 1394-1396, mais sur ce point nous avons quelques réserves méthodologiques $^{31}$.

Pour le $\mathrm{XV}^{\mathrm{c}}$ siècle nous voulons attirer l'attention sur les données militaires de 1411 et 1436, relatives aux mobilisations de la milice brugeoise (comme des autres milices flamandes), auxquelles les ducs de Bourgogne firent appel pour les campagnes contre les Anglais à Ham en Vermandois (1411) et à Calais (1436) $)^{32}$. Pour 1411, il s'agit de nouveau d'un paiement de solde mensuelle (maendghelt), cette fois réparti autrement qu'en 1338. Apparemment le taux de répartition a été adapté à une nouvelle réalité démographique et socioprofessionnelle, reflétant le changement dans la situation économique de la ville de Bruges au $X V^{\mathrm{c}}$ siècle $^{33}$. Les pourcentages relatifs de chaque corps de métier ou section de la bourgeoisie ne diffèrent que légèrement de ceux du voud de 1436, que nous avons découvert dans le registre au renouvellement de la $\mathrm{loi}^{34}$. On ne sait pas comment ces nouvelles répartitions ont

30. - I. DE MEYER, « De sociale structuren te Brugge in de 14de ceuw », in Anciens Pays et Assemblées d'Etats, 54, 1971, p. 7-78. Les divisions professionnelles construites par l'auteur selon des critères douteux, sont critiquées par W. PREvenier, De bevolkingscijfers..., p. 291. Il s'agit des sections de Saint-Nicolas, Saint-Jacques et Notre Dame.

31. - Voir les tables dans I. DE MEYER, « Sociale structuren... », p. 30-32 : elle confond des catégories économiques et juridiques el met des métiers, des professions libérales et des poortersneringen (organisations professionnelles qui n'étaient pas incluses dans le cadre corporatif strict) dans la même liste. Quand ses pourcentages différent légèrement des nôtres, c'est parce qu'elle exclut le membre de la bourgeoisie de la totalité. De plus, W. Prevenier, De bevolkingscijfers..., p. 289, remarque à juste titre que les chiffres que de Meyer retient pour 1379 n'ont aucun rapport avec la réalité, car ils ne représentent qu’une sclérose des chiffres antérieurs. Néanmoins, les conclusions qu'clle tirc pour le XIV siècle restent globalement valables.

32. - C. R. Vaughan, John the Fearless. The Growth of Burgundian Power; London, 1966 et iD., Philip the Good. The apogee of Burgundy, London, 1970, p. 000 ; V. FRIs, « Het Brugsche Calfvel van 1407-1411 », Bulletin de l'Académie d'Archéologie de Belgique, 1911.

33. - L. Gillionis-VAN Si:Virien, Inventaire..., III, p. 131-133.

34. - Archives de la ville de Bruges (AVB), Registers van de Wetsvernieuwingen, 1421 - 1443 (voir VANDFWALL, Beknopte inventaris, p. 68, $\left.\mathrm{n}^{\circ} 114\right)$, fol. $116 \mathrm{v}^{\circ}$ : Dit hier nater es de tax van der poorters ende ambochten hoe vele dat elc gheeft ter voude tweke groot es CCCCL man ende als tghemeen land van Vlaenderen gheheel gheeft MMM man comdt de stede als boven ende zal aldus voord an bliven. Cette liste avait déjà élé éditéc par P. DE STOOP, « Particularités sur les corporations et 
été déterminées ${ }^{35}$. Ce sont probablement le magistrat d'une part et les chefs de chaque zestendeel et les 54 doyens de métiers (assemblés dans le ghemeene buke van der stede ou Grand Conseil de la ville) d'autre part, qui s'en sont chargés. Bien qu'on ait peu de documents qui pourraient nous renseigner sur son fonctionnement, le Grand Conseil semble avoir été convoqué pour donner aux importantes décisions militaires et fiscales - qui touchaient la communauté urbaine dans sa totalité - une autorité plus générale. Surtout durant des révoltes et des crises de pouvoir, le Grand Conseil de Bruges fonctionnait comme autre instrument de gouvernement de la ville ou comme facteur stabilisateur pour le magistrat ${ }^{36}$. Une version d'un témoignage brugeois de la grande révolte de 1436-1438 (le manuscrit 437 conservé à la bibliothèque municipale de Bruges) confirme qu'en 1436 on choisissait des miliciens de la bourgeoisie et de chaque corporation, « chacun selon son transport ${ }^{37}$. Cela implique qu'il y avait une répartition fixe (ou transport, comme nous l'avons vu, un terme également utilisé pour des taux de répartitions fiscales). Compte tenu des petites différences entre les taux du maenghelt de 1411 et du voud de 1436, on peut conclure qu'il y avait à Bruges dans la première moitié du $\mathrm{XV}^{\mathrm{C}}$ siècle, un transport pour la levée de la milice communale. Sans doute fut-il fixé en 1411, quand l'armée urbaine fut alors convo-

34. - (suite) méticrs de Bruges ", Annales de la Société d'Emulation de Bruges. V. 1843. p. 158160, mais d'une manière peu scientifique et sans référence à la source. Cette édition est d'ailleurs passée complètement inaperçue et nous l'avions trouvée plutôt par hasard après avoir découvert l'original.

35. - W. PRFVIENIER, « Bevolkeringscijfers... », p. 285 confirme que, bien que le voud de 1.3.38-1.340 soit plus ou moins comparable avec des versions antérieures de 1302.1316 et 1321-1322, il y a quand même des différences. Ceci implique qu'il y avait des tentatives (de la base? ?) de changer les quotas et que les corporations qui s'étaient accrues après 1322, ont mobilisé davantage en 13381340. On peut imaginer une tendance comparable entre 1379 et 1411 .

36. - W. Bu.ockmans, De volkswertegenwoordiging in het graafschap, Vlaanderen in de overgang van Middeleeuwen natur Nieuwe Tijden, Bruxelles, 1978, p. 84 et 86: J. DuMolyn, De Brugse opstand van 1436-1438... Coutrai-Heule, 1997, p. 164 : le Grand Conscil brugeois est comparable à la Collace gantoise, représentant aussi les élites corporatives, bien que cette dernière institution avait plus de poids dans le processus politique de la ville et surtout un caractère plus permanent (ef. M. BOONE, Gemt en de Bomrgondische hertogen 1385-1453. Een sociad-politicke studie van een statasvormingsproces, Bruxelles, 1990, p. 28-32). Les corps de métiers dans le Grand Conseil brugeois étaient parfois représentés non pas par leurs 54 doyens, mais seulement par les 9 (ou dans d'autres cas 18) des plus importants parmi eux, les corporations étant divisées en 9 membres ou leden (cf. J.A. VA. HotTT1, De geschiedenis van Brugge, p. 312).

37. - J. DIMOLIYN, « De Brugse opstand... ». Un aperçu des principaux évènements de cette révolte dans R. Valcihan, Philip the Good..., p. 79-91. Le manuscrit 437 a été transcrit dans le mémoire de licence inédil de M. R. Dal wi, De Cronicke van Blaenderen, historiografische studie en transcriptice. Gand, 1987. Le témoignage brugeois a été inséré dans cette version (et dans plusieurs autres) de la Flandria Generosa, filiation de chroniques «nationales » flamandes (cf. V. LAMBI:RT, De kronieken van Vlaunderen 1164-1520 : een overzicht met bijzondere aandacht voor hun basis, de " (ienealogia Comitum Filandriae " (Flandria Generosa), thèse de licence inédite, Université de Gand, 1988, p. 87 ; une traduction partielle existe en anglais: ID., Chronicles of Flanders 1200-1500, Gand. 1993); V. FRIS, "Essai d'une analyse des Commentarii sive Annales Rerum Flandricarum de Jacques de Meyere ", in Recueil des travaux publiés par la faculté de philosophie et lettres de 1'Université de Gand, 37, fasc., Gand, 1898, p. 208-222. 
quée pour la première fois depuis 1379-1382, à laquelle époque on utilisait encore la répartition de 1338-1340. Peut-être ces chiffres-là furent-ils encore légèrement adaptés en 1436. On constate par exemple que la bourgeoisie recevait $18,58 \%$ de la solde mensuelle en 1411 , mais qu'elle devait fournir $21,17 \%$ des troupes en 1436 . Nous nous abstenons d'une explication sur ce point, mais il faut quand même indiquer que la croissance relative de la part de la bourgeoisie en 1436 fait diminuer les pourcentages de beaucoup de corporations de manière artificielle, par rapport à une probable évolution réelle entre 1411 et $1436^{38}$.

Le postulat de notre raisonnement est, bien sûr, que ces changements dans le transport militaire à Bruges au commencement du $\mathrm{XV}^{\mathrm{e}}$ siècle, reflétaient en quelque sorte la situation économique réelle. On peut supposer que les corps de métiers comme les tisserands, qui en 1338-1340 devaient fournir 15,8\% des miliciens (autant que la bourgeoisie entière) et qui avaient vu décliner leur importance économique et le nombre de leurs membres dans la deuxième moitié du XIV $\mathrm{XV}^{\mathrm{C}}$ siècle, ont voulu changer ce voud de base, encore utilisé en 1379, puisqu'il les forçait à fournir des hommes armés en disproportion totale avec leur nouvelle taille absolue et relative. D'autre part, on peut facilement imaginer que le pouvoir ou l'influence politique de tel ou tel métier - et surtout de la bourgeoisie - étaient aussi importants pour la fixation du voud et, qu'au contraire, c'était une question de prestige politique pour les tisserands de fournir autant de miliciens. Est-ce que le pourcentage de 3,67\% que ces mêmes tisserands fournissaient en 1411 n'est pas une exagération? Il faut aussi constater que les deux mobilisations de la milice brugeoise de 1411 et 1436, ont été suivies de deux révoltes importantes et d'un parallélisme frappant. La milice urbaine, revenue devant les portes de Bruges, utilisa sa force et sa mobilisation du moment pour soutenir des revendications fiscales et politiques. En 1411, les corps de métiers ont remporté un triomphe en effaçant la réaction de la bourgeoisie dominante après les révoltes et les conspirations manquées des ouvriers du textile de la fin du XIV siècle. Ils récupérèrent leur droit d'auto-organisation et beaucoup de leur influence politique et ils forcèrent le magistrat, entre autres, à supprimer la très impopulaire taxe sur les grains. Les miliciens rentrant à Bruges après l'échec du siège de Calais en $1436^{39}$ ont voulu copier cette action collective de la

38. - Ce qui se montre clairement dans le secteur de la confection et de l'industrie de luxe, où on voit une petite dimunition des chiffres en 1411 et 1436 , bien que, comme on le verra plus loin, d'autres données, surtout qualitatives, donnent l'image inverse.

39. - Les événements de 1411 sont traités de façon exhaustive dans V. Fris, Essai d'une analyse... et J. Dumolyn, De Brugse opstand..., qui donne aussi un aperçu des révoltes brugeoises de la fin du $X I v^{e}$ siècle. Pour le contexte du « renversement des alliances » du traité d'Arras de 1435, qui mena à une campagne militaire de Philippe le Bon contre les Anglais, voir M. R. Thillilismans, Bourgogne et Angleterre..., p. 49-61 et C. A. J. ARMSTRONG, « La double monarchie France-Angleterre et la maison de Bourgogne (1420-1435) », Annales de Bourgogne, 1965, p. 81-112. 
génération précédente. Le résultat fut une guerre civile prolongée et finalement une défaite. La réaction unifiée des classes urbaines supérieures et de « l'appareil de l'Etat » centralisateur du duc Philippe le Bon donna le coup de grâce aux rebelles ouvriers et petits producteurs et à leurs aspirations politiques jusqu' au moment où une nouvelle révolte importante éclata en $1477^{40}$.

Il est donc très probable que dans ces moments de luttes de classe, manifestes ou imminentes, des considérations politiques ont joué un rôle important dans la détermination du nombre que chaque section et corporation devait fournir dans le voud de base. Mais aussi dans des années apparemment paisibles, sans grandes révoltes ni luttes sociales, les dissensions et divisions dans les grandes villes flamandes de la fin du Moyen Age étaient présentes partout, horizontalement et verticalement : entre bourgeoisie et métiers, métiers produisant pour le marché international et « petits métiers », tisserands et foulons, apprentis et compagnons d'une part, maîtres d'autre part ${ }^{41}$ etc. La position de chaque groupe dans la milice urbaine possède une valeur non seulement de prestige, mais est aussi chargée de symbolique et d'identitét+2.

Néanmoins, toutes ces réserves et nuances ne nous empêchent pas d'utiliser ces chiffres de répartition corporative, surtout pour esquisser une comparaison entre le $X I V^{\prime \prime}$ siècle et le début $d u X^{\prime \prime}$ siècle. On les utilisera comme indicateurs relatifs et dynamiques pour illustrer certaines tendances, sur le plan économique et démographique, du plus grand marché de l'Europe occidentale.

Pour l'industrie textile à Bruges, le XIV siècle fut une période de dépression et de spécialisation. A cause du déclin depuis environ 1330 de la « sayetterie » urbaine (un secteur important au XIII" siècle) et la percée des strijpte lakens ( draps rayés », une variété de qualité intermédiaire), la ville devint plus qu'avant dépendante de l'approvisionnement en laine anglaise de haute qualité. Dans le secteur des variétés drapières meilleures marché, Bruges ne pouvait plus faire concurrence à l'industrie rurale et à la production dans les petites villes flamandes. Les tisserands des villages et villes secondaires étaient soutenus par ceux qui s'engageaient dans le commerce des draps et de la laine. A Bruges, on voyait de plus en plus l'intervention de nations de com-

40. - Un document très curieux qui énumère lés revendications des révoltés est édité par J. Di MAI.Y., « Rebelheden ende vergaderinghen. Twee Brugse documenten uit de grote opstand van 14.36-14.38 ». Bullerin de la Commission Rovale d'Histoire. CLXII, p. 297-32.3.

41. - W. Bı.окмАNS. «I a manipulation du consensus. Systèmes de pouvoir à la fin du Moyen Age » dans S. (is:sisini (éd.). Principi e città alla fine del medioever, Pise. 1996, p. 437.

42. - P. ARNADE, « Crowds, banners and the marketplace: symbols of defiance and defeat during the Ghent war of 1452-1453 », Journal of Medieval and Renaissance Studies, 24, p. 486-487; 10., Realms of Ritual. Burgundian Ceremony and Civic Life in Late Medieval Ghent, Ithaca-Londres, 1996. 
merçants étrangers, appuyées par les puissants hôteliers et courtiers brugeois, qui, naturellement, défendaient leurs propres intérêts plutôt que ceux de l'industrie brugeoise. Les dénombrements spécifiques d'hommes valides dans le métier des tisserands démontrent incontestablement le déclin numérique dans la deuxième moitié du XIV ${ }^{e}$ siècle $^{+3}$ :

$\begin{aligned} 1338-1340: & 1016 \\ 1372: & 408 \\ 1379: & 384 \\ 1381: & 343 \\ 1382: & 342^{+4}\end{aligned}$

Les causes traditionnelles sont bien connues : la compétition des centres de production nouveaux, le dépeuplement des marchés par la peste, une diminution et une plus grande dispersion de l'exportation de la laine anglaise et l'essor d'une industrie textile anglaise propre ${ }^{45}$. Dans les années trente du XIVe siècle, environ $31 \%$ de la population adulte et masculine travaillait encore dans le secteur de la draperie ${ }^{+6}$. Une comparaison avec les chiffres de 1411 suggère que l'industrie textile brugeoise était au point le plus bas dans le dernier quart du XIV siècle, ce qui est parfaitement compatible avec les luttes sociales violentes qui marquent cette période. J. Vermaut a calculé les inscriptions au métier des tisserands de laine pour le $\mathrm{XV}^{\mathrm{C}}$ siècle (les sources le permettent depuis 1406). Le facteur le plus sensible pour décrire la conjoncture de cette industrie est le nombre annuel des nouvelles inscriptions d'apprentis qui ne sont pas fils de maîtres. Pour la période 1406-1413 il calcule une moyenne de 1,4 inscriptions par année; 10 pour 1413-1429; et seulement 0,6 pour la période entre 1429 et 1441 (marquée, comme nous l'avons vu, par une crise politique, économique et sociale). Nous pouvons donc parler d'une conjoncture montante entre 1413 et 1429 , suivie par une crise et un déclin rapide aboutissant à une révolte populaire (qui avait par ailleurs aussi d'autres origines). En 1434 lc Kontor Major, chef des hanséates à Bruges, mentionnait dans une lettre " l'anéantissement » des métiers de la draperie et la disparition de plus de la moitié de l'industrie drapière à cause des prix élevés de la

\footnotetext{
43. - L'essentiel de ce paragraphe se base sur J. VerMaur, De texticlnijuerheid in Brugge en op loet platteland in Westelijk Vlaandeen voor 1800$)$ : konjunktuurverloop, organisatie en sociale verhouding. Gand, 1974. Cette thèse de doctorat est malheureusement restée inédite et la draperie brugeoise et son organisation sociale ont jusqu’à présent été trop peu étudiées.

44. - Respectivement J.-F. VirbregGen. Het gemeenteleger..., Bruxelles, 1962. p. 78: G. Espinas et H. PIRFixi:, Recucil de documents relatiss à l'histoire de l'industrie drapiere en Flandre. 19(16. t. I, $n^{\circ}$ 175. p. 584-591: L. Gilliomts-Van Severex, Imeritaire... t. II, p. 383; G. Espinas et H. PIRFANit, Recheil... I. I, n' 178, p. 594-601, nº 179, p. 602-604.

45. - J. Vermaut, De textielnijuerheid..., p. 215.

46. - J. Vermaut, ibidem. p. 213; ce qui est d'ailleurs moins qu'à Gand et Ypres à la même période. $\triangle$ Bruges, il y a toujours eu une structure économique plus différentiée à cause du rôle prépondérant du commerce.
} 
laine $^{47}$. En 1436-1437 seulement 66 sacs de laine anglaise furent transbordés vers Calais, point le plus bas de l'histoire du commerce de la laine anglaise ${ }^{48}$. Les raisons d'une conjoncture meilleure dans les années 1410-1429, sont l'approvisionnement stable de laine anglaise et la stimulation de l'exportation à cause des dévaluations monétaires de 1409,1416 et 1418 . La seule façon pour les grandes villes de réagir à l'industrie rurale croissante était la spécialisation dans des variétés précieuses et luxueuses ${ }^{49}$. La répression de la draperie rurale était déjà inutile au $X V^{*}$ siècle et Bruges ne semble pas l'avoir appliquée systématiquement, sauf par nécessité politique ou quand les métiers d'une petite ville comme Eeclo produisaient des imitations illégales de draps brugeois. Quand même, la crise politique de 1407-1411 était aussi déterminée par des revendications populaires qui voulaient limiter la production rurale et depuis 1429 ces dissensions semblent resurgir ${ }^{50}$. Bruges produisait de nouveaux types de draps de luxe depuis le $x V^{\mathrm{C}}$ siècle, comme les brede et smalle lakens (draps « larges » et « étroits»), les fine lakens et les keurlakens. Le magistrat regroupait depuis 1434 la production de smalle lakens de laine flamande, espagnole et écossaise dans la « nouvelle draperie ». Cette institutionalisation doit être considérée comme une mesure pour rendre ces draps plus attrayants sur le marché international, compenser le chômage causé par le manque de laine anglaise et homogénéiser la production des draps de second choix, une nécessité pour l'exportation"' ${ }^{51}$ En tout cas on peut parler de crises et restructurations importantes, et comme les chiffres le démontrent clairement, aussi d'une diminution relative de l'importance socio-démographique de ce secteur dans l'économie brugeoise. Cependant, en comparant les chiffres des voud de 1338 et 1436, un fait saute aux yeux : c'est qu'en 1338 les proportions de taille des quatre métiers du textile (respectivement tisserands, foulons, tondeurs et teinturiers) était environ 9-6-3-1 ${ }^{52}$. En 1436

47. - C. VON DIR ROPP, Hanserecesse, 1. I. p. 1.3 .3 : dacrate date de neringhe lan der draperie, dae' dit lande und ander lande hiir tumme gelegen bij stach, zee'e medde to nichte ghe't (aussi cité par J. H. MinRo, Wool. Cloth and Gold. The struggle for bullion in Anglo-Burgandian trade, 1.3401.378. Bruxelles-Toronto, 1977, p. 104).

48. - Mide'm, p. 115.

49. - J. VERMALT. De kevtielnijuerheidl... P. 63 el passim. Une appréciation plus générale de cette

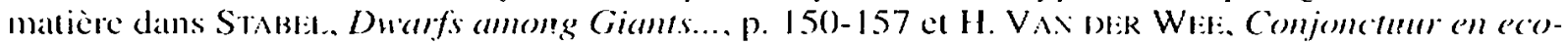
momische groei..., p. 17. Pour les effets des dévaluations voir : H. VAN Wrervike, « De economische en sociale gevolgen van de muntopolitick van de graven van Vlaanderen (13.37-1433.3) ". Anncales de

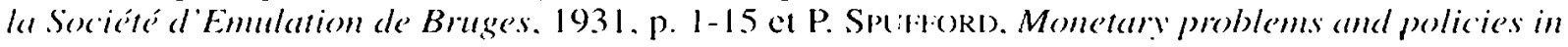
the Burgundian Netherlands. 14.33-1496, Iciden. 1970.

50. - J. De Nol.y, De Brigse opstand.... p. 58-60.

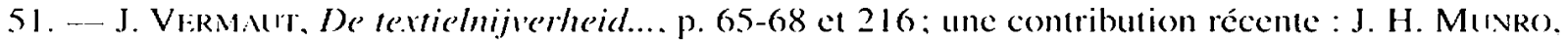
"The Flemish « New Draperies»: The Death and Resurrection of an old Industry, 14th to 17th Centuries", in N. B. HART々 (éd.), The New Draperiés. Oxford, 1997.

52. - Voir aussi les rapports pour Bruges el Gand au XIV" siècle (W. PRI:VienIter. Bevolking.scijfers.... p. 3(2), qui montrent que déjà en 13.38-1340 à Bruges, il y avait plus de tondeurs et teinturiers par rapport au nombre de tisserands, yu’à Gand en 1357. Pour les aspects techniques des différents stades dans la production drapière voir G. DE: POI:RCK, La draperic médiélale en Flamdie et en Arrois. Technicue ef reminologie, 3 t.. Bruges, 1951. 
on calcule une relation d'environ 3-3-3-1. Comment expliquer cette évolution? Le calcul des nouvelles inscriptions pour le métier des tondeurs (pour lequel les sources sont conservées) démontre qu'il était beaucoup moins dépendant de la fluctuation conjoncturelle. Cela est lié à l'approvisionnement régulier à Bruges en tissus ruraux semi-achevés ou inachevés et même en draps anglais, pourtant interdits. Les tondeurs et les teinturiers profitaient beaucoup de la présence de commerçants régionaux et internationaux ${ }^{53}$. Bruges était connu comme un centre important pour le finissage et la teinture de draps : comme les chiffres le démontrent, c'était déjà le cas au XIV siècle (voir la comparaison avec Gand) mais encore plus au $X^{2}$ siècle ${ }^{54}$. Une ordonnance du Hansekontor à Bruges de 1414 indique même que les Hanséates pouvaient faire teindre des draps anglais à Bruges, des produits qui normalement étaient complètement interdits en Flandre et dont ces marchands allemands possédaient seulement le droit de transit ${ }^{55}$. Avec la diminution de l'importance de la production même, tout ceci peut expliquer les chiffres du voud radicalement changés.

Mais il faut quand même se garder de sous-estimer les influences politiques dans la détermination de ces chiffres, notamment pour les métiers de textile aux traditions révolutionnaires. Peut-être les classes dirigeantes voulaient-elles affaiblir leur importance politico-militaire dans la ville et sa milice. Une autre nuance s'impose quand on considère que les hommes valides fournis par le membre de la bourgeoisie étaient probablement, non seulement des marchands et détenteurs d'offices riches, mais tous ceux qui n'étaient pas inscrits dans un corps de métier. Peut-être la main d'oeuvre non qualifiée y était-elle aussi comprise, de même probablement que d'autres qui s'occupaient du textile ou de la confection, mais en dehors des structures corporatives.

Comme nous l'avons vu, à cause du dépeuplement énorme après la révolte, la disette et la peste de 1436-1440, le magistrat brugeois supplia le duc Philippe en 1441 d'ouvrir le droit de bourgeoisie à des tarifs fort réduits : 34 nouveaux maîtres s'inscrivirent au métier des tisserands, 29 chez les tondeurs. A plus long terme, seulement un tiers de ceux-ci demeurèrent maîtres.

53. -.. J. VERMAUT, De textielrijuerheid..., p. 192.

54. - S. Abraham-Thisse, "I Ie commerce des draps de Flandre en Furope du Nord. laut-il encore parler du déclin de la draperie flamande au bas Moyen Age? », in M. Borsil et W. PrI:VIiNIER (éd.). La draperie ancienne des Pass-Bas : débouchés et stratégies de survie (14e-16e siécles). Leuven. 1993. p. 205; voir aussi les règlements dans: Archives de l'Etat à Bruges, Fonds Ambachten (méticrs), $\mathrm{n}^{\circ} 1$ : cartulaire de tous les méticrs brugeois compilé vers 1445. fol. $33 \mathrm{r}^{\circ}$.

55. - W. Brut.r\%, « Engels laken in Vlaanderen in de 14de en 15de eeuw », Annales dé la Société d'Emulation de Bruges. 1971 , p. 21-22. 
Entre 1441 et 1460 cependant, on constate une modeste montée conjoncturelle dans le secteur drapier ${ }^{56}$.

Une industrie de confection comme la bonneterie, pour laquelle Bruges était connue à l'époque, croissait dans cette période de crise. On peut penser que quelques ouvriers drapiers au chômage trouvaient une solution dans cette industrie $^{57}$. La confection était très bien mise en valeur dans un centre commercial où des produits inachevés étaient importés en grande quantité. Entre 1420 et 1432 au moins 32 étrangers immigrèrent à Bruges pour pratiquer la profession de mutsscheerder (qui s'occupaient du finissage des bonnets) ${ }^{58}$. Les tailleurs et couturiers hollandais envahirent Bruges après les hostilités dans leur région en 1433 (quand le duc de Bourgogne y menait une guerre d'annexion) : 36 nouveaux bourgeois pratiquant ce métier, et entre 1433 et 1447 il en arriva encore $22^{54}$. Apparemment ils étaient convaincus de trouver un nouveau travail dans la métropole; 115 tailleurs achetèrent le droit de bourgeoisie entre 1418 et 1447 et le nombre de tapissiers donne une image comparable ${ }^{(1)}$. Les données pour le secteur de la confection nous montrent donc, comme prévu, une toute autre image que celle de la draperie. On voit donc le nombre des tailleurs croître entre 1394-1396 (les chiffres partiels provenant de la taille) et 1411 d'environ $3 \%$ à $6 \%$ pour descendre de nouveau un petit peu en $1436(5 \%)^{(1)}$. Ici les chiffres sont tous beaucoup plus bas et donc moins éloquents que ceux de la draperie, mais globalement on note qu'en 1338-1340 la confection constitue environ un dixième des emplois de la population brugeoise, en 1394-1396 (apparemment?) un huitième. En tout cas, la draperie était un secteur à plus fort coefficient d'emploi, dont les pertes n'étaient pas toutes compensées par l'essor modeste de la confection ${ }^{62}$. Sans doute, la même chose est vraie pour le travail du cuir et la

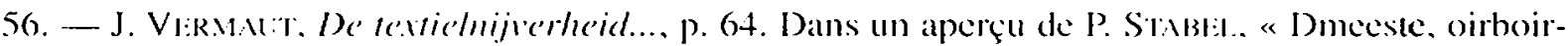
lixste ende proffitelixste let ende neringhe " Een kwantitatieve benadering van de lakenproductic in het latumiddelecuwse en vroegmoderne Vlatanderen. Handelingen rain de Matassheteppij roor

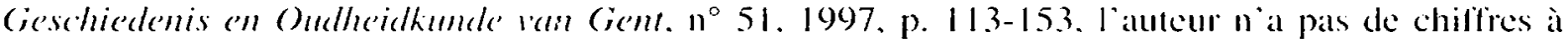
ajouter concernant la production ou la conjoncture dans la draperie brugeoise.

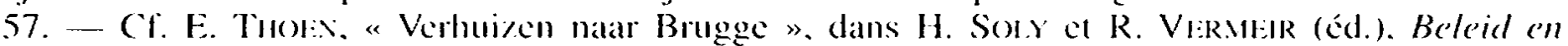
bestumer in de Oude Nedertanden. Liber Amicortum Michel Baclde. 1994. p. 346.

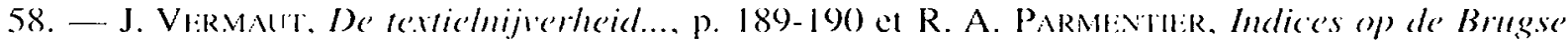
poortersboeken, 1+18-179+. I. I. passim.

59. -- J. VI:RNAMT, ibidem. p. 191-192.

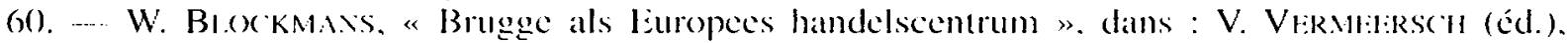
Brugge en Europer. Antwerpen, 1992, p. 46; I1.. «The creative enviremment... ». p. 14, a démontré que les immigrés dans les seckeurs de la confection el l’industrie de luxe élaient déjà très importants alu XIV"siecle.

61. --.- Mais comme indiqué plus hatut, il faut nuancer les différences entre 1411 et 1436, vu que la part de la bourgeoisie monte en tout.

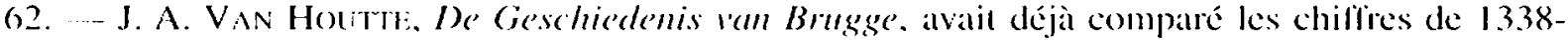
1.340 el 1.394-1.396 en ee qui concerne la confection et l'industrie de luxe. Bien que l'évolution dans les chilfires du roud soit peu éloquente pour ke métier des tisserands de lin, comme il y avalit dauss le deuxième quart du Xv" siècle une hausse de l'exportation de toiles flamandes et vu qu'en 1449 un
} 
pelleterie, des secteurs en expansion en Flandre à cause d'un élevage de bétail plus étendu et stimulé par la consommation croissante de viande et de produits laitiers ${ }^{63}$.

Le Moyen Age tardif vit une importante croissance de l'industrie dite « de luxe »dans les villes et surtout dans des centres commerciaux comme Bruges. La spécialisation et la division du travail dans ce secteur eurent pour conséquence une hausse de la valeur ajoutée. La corporation des peintres se scinda en diverses branches pour les peintres de selles, les peintres de miroirs, les peintres sur draps, les cuirassiers, les peintres sur verre, les parcheminiers et les imprimeurs ${ }^{14}$. La joaillerie brugeoise florissante était fort appréciée en Angleterre ${ }^{65}$. Corroyeurs de cuir noir et de cuir blanc, ceinturiers, zwaardschedemakers («faiseurs de fourreaux»), faiseurs d'arcs et armuriers, selliers, imagiers, orfèvres et faiseurs de chapelets représentaient en 1338 $5,93 \%$ et 6 à $7 \%$ de la population active en 1394-1396. En 1411 on leur paye ensemble environ $11 \%$ de la solde mensuelle et les mêmes corporations que I. de Meyer inclut dans la catégorie « industrie de luxe » en 1394-1396 forment en 1436 ensemble presque $10 \%$ de la milice urbaine ${ }^{6 t}$. Bien sûr, ces chiffres ne nous étonnent pas. Une demande croissante dans les Pays-Bas à cause de la présence de la cour bourguignonne et sa pompe fastueuse, du pouvoir d'achat accru des classes supérieures et de la demande étrangère, stimulait évidemment l'industrie de luxe ${ }^{67}$. A Bruges il faut y ajouter l'énorme pouvoir d'achat qu'avaient les nations étrangères, comme en témoignent les tableaux de Van Eyck.

Une exception semble avoir été le métier des « paternôtiers » (faiseurs de chapelets), une activité industrielle pratiquée seulement à Lübeck et à

62. - (suite) hôtelier brugeois et un marchand anglais échangèrent de grandes quantités de toiles flamandes contre des draps anglais, on pourait supposer que si Bruges n’était pas clle-même un centre de production de toiles (ce qui élait plutôt une industric rurale typiçue), c"était peut-être un centre de finissage avant l'exportation (cf. M. BOON1: « Les toiles de lin des Pays-Bas bourguignons sur le marché anglais (fin XIV"-XV" siècles) ». Publications du Cemtre Européen des Etudes bourguignonnes, 1995, p. 61-82).

63. - R. VAN UYTVI:N, « I a Flandre et le Brabant... », p. 97.

64. - R. VAN UytVEN, « Splendour or Wealth... », p. 109 ; voir aussi J. II. MLiro, « Economic depression and the arts in the fifteenth-century Low Countries ", dans ID., Textiles, Towns and Trade. Essays in the economic history if late medieval England and the Low Conntries. Great Yarmouth, 1994, p. 235-250: voir aussi W. Bı.ockMANs, « The creative Environment... » p. 1.3-14.

65. - M.-R. Thilitimais, Relations commenciales..., p. 240.

66. -. Même s'il élait encore possible que les 3 zestendelen pour lesquelles les registres d’impôts indirects sont conservés pour les amnées 1394-1396, connaissaicnt une plus haute densité en industric de luxe que dans l’autre moitié de la ville, en tout cas le redoublement de lcur délégations militaires en un siècle reflète unc augmentation dimportance significative.

67. - R. VAN UYTVIix, «Splendour or wealth... », p. 101-105; M. P. J. MARTE.S. Arlistic Patronage in Brages Institutions, ca. 1440-1482. (dissertation inédite Ph. D. de l'Université de California, Santa Barbara. 1992). 
Bruges. En 1420, on estimait le nombre de maîtres «paternôtiers » encore à 70 , mais au cours du $\mathrm{XV}^{\mathrm{e}}$ siècle il semble avoir reculétix. Les armuriers, pas encore présents dans le voud de 1338, étaient assez bien représentés, pour un petit métier spécialisé, en 1411 et 1436. Peut-être cette donnée correspondelle au développement d'une industrie d'artillerie relativement importante à Bruges au $X V^{\mathrm{e}}$ siècle ${ }^{(1)}$.

Mais, aussi bien que dans le secteur de la confection, la croissance de l'industrie de luxe ne pouvait pas compenser les pertes d'emploi dans la draperie. Le fait que le nombre moyen de nouveaux bourgeois continue à baisser dans la deuxième moitié du $X^{2}$ siècle peut probablement être lié à l'augmentation de l'importance relative du secteur de luxe dans l'économie, un secteur à moins fort coefficient d'emploi ${ }^{70}$. Bien que Bruges fût renommée pour son industrie du livre florissante, la ville ne comptait que 16 miniaturistes en 1427, et environ 50 scribes, libraires et parcheminiers ${ }^{71}$. Pour un tisserand ou foulon c'était d'ailleurs presque impossible d'opérer une « reconversion professionnelle » et d'entrer dans les métiers de luxe, qui avaient un caractère plutôt fermé et népotiste et qui demandaient tous une formation spécialisée.

\section{Essai de calcul de la population en 1436-1440}

A cause des rapines des Anglais en Flandre occidentale après la défaite de Calais en 1436, de la révolte de Bruges de 1436-1438 (qui avait attiré beaucoup de partisans dans les villages et petites villes aux alentours), d'une guerre avec l'Ecluse, avant-port de Bruges qui cherchait à éroder les privilèges de son chef-lieu, provoquant encore plus de destructions dans la campagne, et à cause de quelques mauvaises récoltes, la disette de 1438 se transforma à Bruges en une véritable famine. La population ne pouvait plus faire face à la nouvelle catastrophe : une épidémie de peste sévère, la pre-

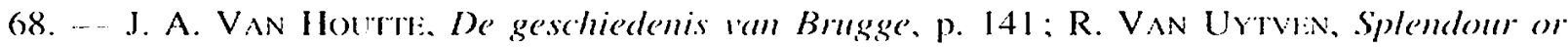
"realth... graph. 1 : les artisans brugeois employés dans les secteurs de textile. meubles et industrie de luxe 1.302-1379-1490.

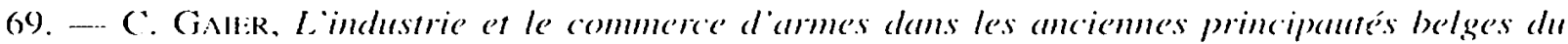
xur ì la fin du $x \mathrm{v} r$ siécle. Paris. 1973. p. 117, 124.

70. - J. A. VAN HolTtri. De geschiedenis ran Brugge, p. 156 : dans le années 1440, 260 nouveaux bourgeois s’inscrivent suite à l'appel du magistral et à la réduction de la taxe après la catastrophe démographique suivant la révolte de 1436-1438. Ce nombre baisse graduellement dans les décennies suivantes : respectivement 163,153.126.98 et 39 par an.

71. - R. VAN UYTVti., « Splendour or wealth... ". p. 110 . L auteur conclut son étude par ce bon mot gue pour beaucoup de villes des Pays-Bas la " toison d'or" de Philippe le Bon n’était qu" une reproduction misérable de la toison de laine des générations antérieures...

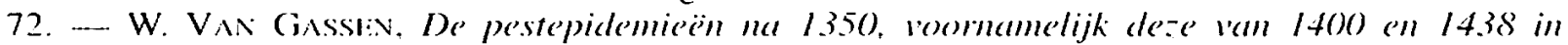
Vlaumderen en Henegounen, thèse de licence inédite. Université de Gand. 1952 et W. P. Bi.ockmass, "The social and economic effects of Plague in the Low Countries 1.349-1500)». Re'lle Belge de Philologie et Histoire, 1980), p. 851-853; par inadvertance P. Stabla. De kleine studl... p. 67 s'est apparemment trompé en faisant mention d'une épidémie de peste à Bruges en 1420-1421; pour laquelle il ne donne aucune source. 
mière en Flandre depuis 1400-140172. La chronique erronément attribuée à Jan Van Dixmude, autre version du récit d'un témoin de la révolte brugeoise, énumère les morts comptés par paroisse et prétend qu'en octobre 1438 un cinquième de la population était morte ou avait quitté la ville à cause de la crise économique et parce qu'ils ne pouvaient plus payer leurs dettes. On a aussi noté que les revenus de la taxe indirecte (ou assise) sur la bière, denrée de demande immuable, avaient diminué d'un tiers après l'année fiscale 1438$1439^{73}$. Selon Van Gassen, dans la période suivant la révolte, la population brugeoise diminua d'un tiers ou d'un quart ${ }^{74}$.

Pour payer l'amende énorme que le duc Philippe avait imposée à sa ville désobéissante, le magistrat dut entre autre organiser la levée d'impôts directs, qu'on appelait pointinghen. Un seul des registres de cette taxe est conservé, celui de la section de Saint-Jean en 1440-1441. Il s'agit d'un cahier qui donne la liste de 855 redevables, probablement des chefs de famille ${ }^{75}$. Dans le taux de répartition de la milice urbaine en 1436, la section Saint-Jean ne devait fournir que $15,3 \%$ des miliciens de la bourgeoisie ${ }^{76}$. Il faut bien noter qu'il s'agit ici seulement des bourgeois qui n'étaient pas inscrits dans un corps de

73. W. Bl_ockmans, p. 857 ; Chronique de Jan Van Dixmude édité par J. J. DF S.MET, in Corpus Chronicorum Flandricie, Bruxelles, 1856, t. III, p. 103. A cause de la façon changeante de la collection des assises de la bière au $x V^{\prime}$ siècle, il n'y a que quelques années pour lesquelles on pourrait utiliser ces chiffres comme indicaleur de la demande réelle et il serait impossible de donner des résultats séricls. E. THOEN, Landbouweconomic..., p. 47-48, table 1, démontre que dans les années 1439-1440, il y eut aussi une grave crise de mortalité dans le sud du comté, région d'ailleurs sans doute moins frappée par les événements politique turbulents de 1436-1437. D'autre part, l'autcur minimise la signification démographique de cette crise (p. 158). Bien qu'il ait globalement raison de relativiser l'impact de cetle crise à plus long terme (étant donné çu'elle ne faisait qu'interrompre la hausse démographique séculaire en Flandre de ca. 1400-ca. 1470) et de mettre les historicns économiques en garde de n'étudicr l'évolution démographique qu'avec des crises de mortalité, nous estimons néanmoins que ses conséquences néfastes furent beaucoup plus prononcées à Bruges et dans la Flandre maritime. Les pertes catastrophiques dans la production agricole causées par les pillages des Anglais dans la région au nord-est de Bruges en 1436 ont été étudiées par I. M. VAN WERVEKF, « De Fngelschen in de ambachten van Oostburg en Ijzendijke in 1436 », Annales de la Société d'Emulation de Bruges, 1931, p. 183-188, et l'année suivant voyait aussi d'importants mouvements de bandes armées de rebelles ou ducales, cl. J. Duvor.y. De Brugse' opstand, passim.

74. - W. VAN Gassen. De pestepidemiënn..., p. 56-70. L’auteur utilise toute les données quantifiables, mais ses arguments reposent principalement sur la chronique mentionnée plus haut, qui semble donner une impression digne de confiance, énumérant les morts dans les diverses paroisses pendant des intervalles de temps: W. BLOCKMANS, «Die Niederlande vor und nach 1400 : eine Gesellschaft in der Krise?" ", dans F. Shint et W. Eberhard (éd.), Europa 1400. Die Krise des Spätmithelalters, Stuttgart, 1994, qui appelle les annécs 1437-1439. une allgemeine Sterbekrisen et des allgemein-europäischen Krisenjahre, préfère une diminution d'un cinquième ou d'un sixième. pourtant sans donner d'arguments nouveaux : effectivement, le chroniqueur Van Dixmude avait parlé d'une diminution d'un cinquième, mais ceci plus d'une année avant la fin de la catastrophe. 75. - Archives Communales de Bruges, comptes de la ville, annexes (A. VANDFwal.l.F, Beknopte inventaris..., Decl I. Oud Archief, p. 208); voir aussi L. GillodTs VA.x SEveren, Inventaire..., t. V, p. 250-210.

76. - CI. la table. 
métier. Ces derniers avaient leur propre quota de mobilisation. On ne sait donc pas si ce $15,3 \%$ reflète l'importance relative des sections ou plutôt une différence dans la concentration de métiers et bourgeoisie dans ce quartier. En tout cas, la multiplication par le coefficient $6,54(=100 / 15,3)$ est moins théorique que simplement par six. Ainsi on atteint un total hypothétique de 5592 chefs de familles à Bruges. Comme nous l'avons vu, le facteur 5 est préférable pour une ville comme Bruges. D'autre part, après une période de disette et d'épidémie intense, on peut présumer une mortalité infantile plus élevée, de sorte que le facteur 4 paraît préférable. Faisons les deux calculs pour la population de Bruges en 1440-1441 :

$$
\begin{aligned}
& 5592 \times 4=22368 \text { (minimum) } \\
& 5592 \times 5=27960 \text { (minimum) }
\end{aligned}
$$

Si nous suivons la conclusion/hypothèse de Van Gassen que durant la crise de mortalité d'environ 1438-1440 la population avait diminuée d'un tiers ou d'un quart, nous obtenons les résultats suivants pour Bruges, disons en 1436, avant le début des misères :

$$
\begin{array}{ll}
1440 & 1436 \\
22368+50 \%= & 35052(\text { minimum) } \\
27960+50 \%= & 41940 \text { (maximum) (un tiers) } \\
1440 \quad & 1436 \\
22368+33,3 \%= & 29827 \text { (minimum) } \\
27960+33,3 \%= & 37280 \text { (maximum) (un quart) }
\end{array}
$$

Nous laissons ici de côté tous les arguments sur le nombre de clercs, étrangers, pauvres etc., qui ont déjà été amplement formulés plus haut, pour proposer une simple estimation de 35000 habitants avant la crise démographique et 25000 après, en effaçant toutes les nuances et les subtilités, si importantes qu'elles soient, il faut bien s'en rendre compte ${ }^{77}$.

En récapitulant, peut-on dire que le $X V^{c}$ siècle n'a pas du tout signifié un déclin pour Bruges sur le plan démographique? La population brugeoise suit

77. - Compare\% avec d autres chiffres pour le milicu du $x v^{*}$ siècle : Cologne : $300(0)$; 1 übeck : 25000 : Danyig : $20000($ W. B1.06 KMn.s. « Princes conquérants et bourgeois calculateurs. I c poids des réseaux urbains dans la formation des états », dans N. Bul.st el J.-Ph. Gisilit (éds). La rillé, la

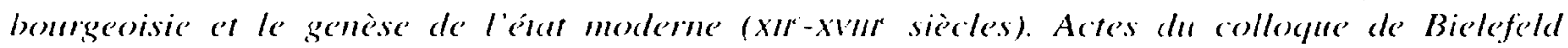

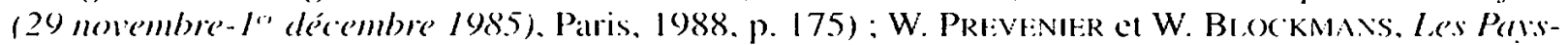
Bas bounguignons..., p. 28-45: une population à Anvers de 15000 personnes en $1437 \mathrm{et} 3900() \mathrm{en}$ 1526: P. BNIROCH. J. BNTOU et P. Cin:VRl: La population... (sans prolonde critique de source et done de valcur très inégale), p. 16: Barcelone 1400:38000 et 1500:20000: p. $27:$ Lille $1400: 22000$ \& 1500: $26000:$ Lyon 1400:33000 el 1500:50000: p. 33: Londres 1400:45000) et 1500): $50000:$ p. $43:$ Florence $1400: 55000$ et $1500: 55000:$ p. $53:$ I eyde $1400: 6000$ et $1500: 14000$. 
plus ou moins le mouvement conjoncturel du prétendu « modèle économique bourguignon ${ }^{78}$. Une période noire pour l'économie flamande dans le dernier quart du XIV siècle provoquée par le déclin de la production agricole et industrielle (surtout la draperie classique), une réduction des débouchés sur les marchés internes et externes, et finalement - liés à la contraction de I'industrie et du commerce - des révoltes et une guerre civile désastreuse en $1379-1385^{70}$. Nous pouvons supposer que cette période noire se termina après l'épidémie de peste de 1400-1401 et que la population brugeoise pouvait de nouveau croître, jusqu'au niveau d'environ 35000 personnes en $1436^{8 *}$. Comme nous l'avons décrit, le nombre de la population reçut un coup sérieux dans les années suivantes et diminua jusqu'à environ 25000 personnes. Comme le magistrat l'écrivait en 1440, Bruges était grandement appouvry et forment desminué de peuple; une politique populationniste fut jugée nécessaire et les tarifs pour acheter le droit de bourgeoisie furent réduits ${ }^{81}$. Mais bien que les conséquences de la révolte de 1436-1438 aient atteint gravement les finances publiques de la ville, la croissance économique des années cinquante et soixante du $X V^{\mathfrak{c}}$ siècle — l'âge d'or « bourguignon » - a de nouveau fait augmenter le nombre des Brugeois jusqu'à environ 40000 en $1477^{82}$. Trois décennies ont suffi pour largement compenser les effets d'une

78. - R. VAN UyTVEN, « Ial Flandre et le Brabant... », p. 302 et 316 ; H. VAN DIik Wial, «Conjunctuur en conomische geschiedenis in de Zuidelijke Nederlanden tijdens de $14 \mathrm{e}$, $15 \mathrm{e}$ en 16e eeuw. Crisistypologie en structuurwijziging », Historische Aspecten van de economische groci, Antwerpen, 1972, p. 100-128; II)., « Industrial dynamics and the process of urbanization and deurbanization from the late middle ages to the eightecnth century » dans ID. (éd.), The Rise and Decline of Urban Industries in Italy and the Low Countries (late Middle Ages-early Modern Times), Leuven, 1988, p. 307-381.

79. - W. P Blockmans, G. Pieters, W. Prevenier et R. W. M. Van Schaïck, « Tussen Crisis en Wclvaart... », p. 59 ; A. VERHLIST, «L'économie rurale de la Flandre et la dépression économique du bas Moyen Age ", Etudes Rurales, 1963, p. 68-80; E. THOEN, Landbouweconomie...

80. - Pour la remonté démographique générale à cette période voir : ibidem, p. $155-160$ et 229 : W. BLOCKMANS, «Die Niederlande vor und nach 1400... », p. 122 : in diesen Zeitraum gab es einen langsamen Bevölkerungsauwachs und einen Stillsland der Reallöhne. Notez encore bien l'importante différence avec l'évolution générale de la population française, où presque partout, on constate un effondrement dans la première moitié du siècle, sauf par exemple dans les villes bretonnes, où la croissance de la fin du XIVe siècle s'est poursuivie jusqu'à environ 1430-1450 (A. HIGOUNET-NADAL, Population.... p. 198-199).

81. - Archives Communales de Bruges, Registres de bourgeois, tome 1434-1449, fol. $23 r^{\circ}-24 v^{\circ}$. Le duc permettait la ville d'ouvrir la bourgeoisie pour chacun pour le petit tarif de $5 \mathrm{~s}$. gros monnaic de Flandre et $20 \mathrm{~s}$. gr pour la corporation dans laquelle on voulait entrer. On cherchait donc clairement des immigrés productifs. Pour les effets de cette ordonnance voir E. THOEN, « Immigration to Bruges... », passim. Exemples de villes françaises (Foix et Périgueux) qui ont aussi mené une politique de peuplement dans A. Higounet-NaDAl, Population..., p. 203.

82. - R. VAN UYTVFN, «I a Flandre ef le Brabant... », p. 302 sille la conjoncture montante entre 1442 et $1470 / 1475$; J.-P. Sosson, Les travaux publics..., p. 226 constate aussi une montée dans les salaires réels.

83. - On a calculé pour le Hainaut que les pertes démographiques causées par la peste de 1400 1402 étaient quasiment effacées dans les 35 annécs suivantes (W. BlockMANS, « Tussen Crisis en Welvaart...", p. 59). 
grave crise de mortalité ${ }^{8 ?}$. Depuis les années 1470, l'évolution négative du nombre moyen de nouveaux bourgeois accentuait la conjoncture en baisse ${ }^{8-4}$. La réduction des tarifs de droit de bourgeoisie en 1496 ne pouvait plus stimuler la croissance démographique comme elle l'avait fait en $1441^{85}$. Il était déjà trop tard pour un tel volontarisme.

\section{Epilogue et conclusions}

Les commentaires de la fin du $X V^{\mathbb{C}}$ siècle donnent une image déjà beaucoup plus négative. L'empereur Maximilien parle en 1493 de la dépopulation de Bruges, du départ des marchands étrangers et de la désolation de la campagne dans les alentours de la ville ${ }^{86}$. A. Wyffels a calculé la population de Bruges pour l'époque moderne ${ }^{87}$. Il cite une lettre de Maximilien d'Autriche et Philippe le Beau du 11 décembre 1494 à la ville de Bruges :

Quatre a cinq mil maisons, toutes vagues, closes et venans en ruyne... grand nombre de bourgeois et habitans qui souloient demourer en la dicte ville l'ont habandonnee $e^{\text {xx }}$.

En 1480-1482 et en 1522 la ville de Bruges avait organisé un dénombrement de la population masculine pour estimer les besoins dans ces années de famine. Au $X V r^{\circ}$ siècle cela était devenu une pratique courante, mais les sources qui auraient pu nous fournir les résultats sont malheureusement perdues $^{89}$. R. Mols a utilisé le register van zestendeelen, une source fiscale, dans laquelle on trouve une liste de 8129 parcelles intra muros, pour atteindre un minimum de 40000 habitants en $1580^{\%}$. Wyffels rejette ce chiffre comme trop osé. On ne sait pas combien de ces parcelles étaient effectivement habi-

\footnotetext{
84. - Cl. E. Thol: Landbouweconomie..., p. 337 ; cependant selon nous, l'auteur se trompe en laissant commeneer cette tendance négative en 1460; comme ses propres chiffres (voir table) le démontrent clairement, la rupture principale se trouve entre les décennies 1460-1469 et 147)-1479, ce qui correspond d'ailleurs mieux à l'état actuel de notre connaissance des mouvements conjoncturels aux Pays-Bas pendant l'époque bourguignonne. Comme le même auteur a déjà démontré que dans la campagne le sommet de la croissance économique et démographique était déjà dépassé après 1450 (E. THOEN, Landbotureconomie..., p. 229), il y avait peut-être un certain décalage campagneville.

85. - Ibidem, p. 340.

86. -- R. VAN UYTVEN, Stages of economic decline.... p. 262. A. JANSSENS, p. 32 reproche à R. VAN UYTVEN, "Plutokratie in de oude democratieën der Nederlanden », Handelingen van de koninklijke Zuidhederlandse Maatschappij voor Taal, Letterkunde en Geschiedenis, XVI, 1962, p. 389, n. 5, de supposer a priori une population d'environ 30000 personnes à la fin du $X v^{\prime \prime}$ siècle. Mais il est très possible qu'il y ait un tel écart entre $1477 \mathrm{et}$, par exemple, 1497 ( la lin » du Xv" siècle), après deux décennies de guerres civiles et de destructions dans la campagne, cf. R. VaN UYTVEN, « Politick en ceonomic : de crisis der late $\mathrm{Xv}^{\mathrm{c}}$ ecuw », Revue Belge de Philologie et d'Histoire. 1975. p. 10971149.

87 - A. WrFFELS, "De omvang en evolutie van het Brugse bevolkingscijfer in de 17 de en de 18 de ecuw » Revue Belge de Philologie et d'Histoire, 1958, p. 1243-1274.

88. - A. WYFFELS, ibidem, p. 1244 ; L. GIl.lodOtS-VAN SiVIEREN, Inventaire..., t. VI, p. 386.

89. - A. WYHFILS, ibidem, p. 1245-1246.

90. - R. Mol.s, Introduction à la démographie..., t. II, 195, p. 522.
} 
tées et tenant compte des protestants émigrés, il parvient à un chiffre de 26000 à 27000 Brugeois à la fin du XVI $I^{c}$ siècle ${ }^{91}$. L'historien du XVIII' siècle J.-P. Van Male cite les résultats d'un dénombrement pour l'année 1699 : 35156 habitants. Celui de 1748 a été conservé et donne le chiffre de 27821 personnes. En mai 1784, la population brugeoise comptait 30846 personnes, d'après les données fournies par un recensement du gouvernement autrichien. Dans un calcul élaboré, Wyffels affine ces chiffres, utilisant les registres paroissiaux et des status animarum conservés pour quelques paroisses brugeoises ${ }^{92}$.

La fin du Moyen Age forme donc une rupture claire sur le plan démographique. Une conclusion très peu étonnante, il est vrai, mais on a définitivement démontré que la population du $\mathrm{XV}^{\mathrm{e}}$ siècle fluctuait encore autour des mêmes chiffres qu'au XIV ${ }^{c}$ siècle. Durant cette époque des crises de mortalité étaient suivies par des périodes de redressement, dans lesquelles l'immigration était un facteur important. Néanmoins, la draperie n'a pas conservé la même importance qu'aux $\mathrm{XIII}^{\mathfrak{c}}$ et $\mathrm{XIV}^{\mathrm{c}}$ siècles et n'était probablement pas compensée en nombre d'emplois par les « nouvelles draperies », la confection ou l'industrie de luxe. La césure de l'an 1500 (ou sans doute plus exactement la période 1480-1500) est moins grave quant au nombre d'habitants qu'à la perte d'une très grande partie de sa fonction commerciale que la ville dut subir ${ }^{93}$. Bien que Zeger Van Maele, Brugeois du XVI ${ }^{\mathfrak{c}}$ siècle, ait beaucoup déploré la ruine du port mondial d'autrefois ${ }^{94}$, sa ville natale continuait à exercer beaucoup de fonctions urbaines d'importance régionale, comme ville provinciale mais toujours vivante ${ }^{95}$.

Jan DUMOLYN

Jan Dumolyn, assistant au département d'histoire médiévale de l'Université de Gand, Blandijnberg 2, B-9000 Gent.

91. - A. WYIIILLS, « De omvang en evolutie... », p. 1244-1246.

92. - Ibidem, p. 1257.

93. - La situation de Bruges correspondait d'ailleurs à la tendance démographique générale en Flandre entre la fin du $\times v^{e}$ et les premières décennies du $x v I^{*}$ siècle. Bruges avec sa châtellenie (le «Franc de Bruges ») et ses petites villes satellites formaient une région particulièrement frappée par cette crise, cf. P. SrAB1:L, « De-urbanisation and Urban Decline in Flanders from 1500 to $1800:$ The Disintegration of an Urban System? ", dans D. MC CABI (éd.), European Urbanisation, Social Structure and Problems between the eighteenth and twentieth Century, Leicester, 1995, p. 87-108. 94. - A. Diwittr el A. Viarine (éd.), De lamentatie van Zeghere van Male. Brugge na de opstand tegen Spanje, 1590, Brugge, 1977.

95. - Bruges maintint son niveau pendant l'époque moderne principalement grâce à l'étape de la laine espagnole (J. VAN HoutTE, De Geschiedenis van Brugge, p. 430), et restait un centre financier au commencement du XVI" siècle : BRULEZ, Brugge en Antwerpen. 
Section de la ville ou corps de métier

Saint-Jean

Saint-Donatien

Notre Dame

Saint-Jacques

Saint-Nicolas

Carmes

Total de la bourgeoisie

Tisserands

Foulons

Tondeurs

Teinturiers

Bouchers

Poissonniers

Charpentiers

Maçons

Couvreurs de tuile

Plombiers

Plâtriers

Couvreurs de paille

Scieurs

Jaugeurs de vin

Déchargeurs de vin

Tonneliers

Charrons

Tourneurs

"Escriniers" (menuisiers)

Imagiers et selliers

Archiers

Cordiers

Potiers

Maréchaux

Orfèvres

Armuriers

Batteurs d'étain

Cordonniers

Corroyeurs de noir cuir

Tanneurs

"Adobeurs " (corroyeurs de cuir espagnol)

Boursiers et corroyeurs de blanc cuir

Gantiers

Chaussetiers

Tailleurs

Courte-pointiers

“Agneliers» (pelletiers)

«Vieuwariers " (fripiers)

Pelletiers de vieux

"Sauvaginiers" (pelletiers)

Boulangers

Meuniers

Chapeliers

Tapissiers

Tisserands de toile

Batteurs de laine

Barbiers

Ceinturiers

Gaîniers

Faiseurs de chapelets

Courtiers

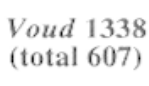

$\%$

2,1
Maandgeld 1411

(Ib. gros Flamand)

(123 lb. 5 s. 9 d.)

3 lb. 12 s. 4 d.

3 lb. 12 s. 4 d.

4 lb. 4 s. 5 d.

4 lb. 4 s. 5 d.

3 lb. 18 s. 5 d.

3 lb. 6 s. 4 d.

22 lb. 18 s. 3 d.

4 lb. 10 s. $6 \mathrm{~d}$.

4 lb. 10 s. 6 d.

4 lb. 10 s. 6 d.

l lb. 16 s. 2 d.

4 lb. 10 s. 6 d.

2 lb. 8 s. 3 d.

$6 \mathrm{lb} .6 \mathrm{~s} .8 \mathrm{~d}$.

4 lb. 4 s. 5,5 d.

$11 \mathrm{lb}, 4 \mathrm{~s}, 1,5 \mathrm{~d}$.

0 lb. 6 s. 0 d.

0 lb. 6 s. $0 \mathrm{~d}$.

0 lb. 6 s. 0 d.

0 lb. 12 s. 0,25 d.

1 lb. 4 s. 1,5 d.

0 lb. 12 s. 0,25 d.

I lb. 4 s. $1,5 \mathrm{~d}$

0 lb. 12 s. 0,25 d.

1 lb. 4 s. $1,5 \mathrm{~d}$.

1 lb. 4 s. 1,5 d.

2 lb. 2 s. 2,25 d.

0 lb. 18 s. 1 d.

0 lb. 6 s. $0 \mathrm{~d}$.

0 lb. 6 s. 0 d.

4 lb. 16 s. 6 d.

$1 \mathrm{lb} .16 \mathrm{~s} .6 \mathrm{~d}$.

0 lb. 18 s. 1 d.

0 lb. 18 s. 1 d.

4 lb. 4 s. 5,5 d.

0 lb. 18 s. 1 d.

2 lb. 14 s. 3,5 d.

0 lb. 24 s. 1,5 d.

$11 \mathrm{lb}, 4 \mathrm{~s}, 1,5 \mathrm{~d}$.

0 lb. $18 \mathrm{~s} .1 \mathrm{~d}$.

0 lb. 6 s. 0 d.

7 lb. 4 s. 9,5 d.

0 lb. 20 s. 2 d.

$0 \mathrm{lb}, 18 \mathrm{~s} .1 \mathrm{~d}$.

2 lb. 14 s. 3,5 d.

0 lb. 30 s. 2 d.

0 lb. 30 s. 2 d.

3 lb. 12 s. 4,5 d.

1 lb. 4 s. $1,5 \mathrm{~d}$.

0 lb. 6 s. 0 d.

0 lb. 6 s. $0 \mathrm{~d}$

2 lb. 2 s. 0,25 d.

0 lb. 18 s. 1 d.

1 lb. 4 s. $1,5 \mathrm{~d}$.

0 lb. 18 s. 1 d.

0 lb. 6 s. 0 d.

2 lb. 16 s. 2 d.

9 lb. 13 s. 0,25 d.

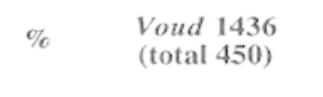

$\%$

$\begin{array}{lll}2,93 & 15 & 3,33\end{array}$

$\begin{array}{ll}2.93 & 15\end{array}$

$\begin{array}{lll}3,42 & 19 & 4,22\end{array}$

$\begin{array}{lll}3,42 & 19 & 4,22\end{array}$

3,56

3,11

21,17

3,11

3,11

3,11

1,11

2,89

1,11

4,44

2,44

0,89

0,22

0,22

0,22

0,67

0,89

0,44

2,44

0,44

0,89

1,33

0,89

0,89

0,22

0,22

3,56

1.33

0,67

0,67

3,11

0,67

1,56

0,67

0,89

0,89

0,22

4,89

1,11

0,67

1,56

1,11

1,11

2,67

0,89

0,22

0,22

1,33

0,67

1,56

0,67

0,22

0,89

7,11

\section{La milice brugeoise 1338-1411-1436.}

N.B. :

- En 1338, 96 bourgeois étaient mentionnés dans le voud. La répartition sur les différentes sections est en fait celle de 1378-1379.

- En 1338. les boursiers el les corrnyeurs de blanc cuir ne formaicut pas encore un seul métier : ils fournissaient chacun 4 miliciens et nous avons aussi fait le total pour pouvoir comparer.

- I ce chiffre de 6 miliciens des gaîniers en 1338 inclut aussi ceux des ceinturiers el faiscurs de chapelets : apparemment ces trois métiers formaient encore au XIV" siècle une unité militaire. ce qui n était plus le cas all $\times v^{\prime \prime}$.

- Là où il n`y a pas de chiffre pour 1338, on peut supposer qu’à l'époque ces métiers (selliers. armuriers, cordiers) n'existaient pas encore de façon autonome. 